Portland State University

PDXScholar

Electrical and Computer Engineering Faculty

Publications and Presentations

Electrical and Computer Engineering

4-2012

\title{
Bayesian Geoacoustic Inversion Using Wind-Driven Ambient Noise
}

Jorge E. Quijano

University of Victoria

Stan E. Dosso

University of Victoria

Jan Dettmer

University of Victoria

Lisa M. Zurk

Portland State University, zurkl@pdx.edu

Martin Siderius

Portland State University, siderius@pdx.edu

See next page for additional authors

Follow this and additional works at: https://pdxscholar.library.pdx.edu/ece_fac

Part of the Electrical and Computer Engineering Commons

Let us know how access to this document benefits you.

\section{Citation Details}

Quijano, J. E., Dosso, S. E., Dettmer, J., Zurk, L. M., Siderius, M., \& Harrison, C. H. (2012). Bayesian geoacoustic inversion using wind-driven ambient noise. Journal Of The Acoustical Society Of America, 131(4), 2658-2667.

This Article is brought to you for free and open access. It has been accepted for inclusion in Electrical and Computer Engineering Faculty Publications and Presentations by an authorized administrator of PDXScholar. Please contact us if we can make this document more accessible: pdxscholar@pdx.edu. 


\section{Authors}

Jorge E. Quijano, Stan E. Dosso, Jan Dettmer, Lisa M. Zurk, Martin Siderius, and Chris H. Harrison 


\title{
Bayesian geoacoustic inversion using wind-driven ambient noise
}

\author{
Jorge E. Quijano, ${ }^{\text {a) }}$ Stan E. Dosso, and Jan Dettmer \\ School of Earth and Ocean Sciences, University of Victoria, 3800 Finnerty Road, Victoria, British Columbia \\ V8P 5C2, Canada
}

Lisa M. Zurk and Martin Siderius

Northwest Electromagnetics and Acoustics Research Laboratory, Portland State University, 1900 SW

4th Avenue, Portland, Oregon 97201

Chris H. Harrison

NATO Undersea Research Centre, Viale San Bartolomeo 400, 19126 La Spezia, Italy

(Received 25 August 2011; revised 24 January 2012; accepted 27 January 2012)

\begin{abstract}
This paper applies Bayesian inversion to bottom-loss data derived from wind-driven ambient noise measurements from a vertical line array to quantify the information content constraining seabed geoacoustic parameters. The inversion utilizes a previously proposed ray-based representation of the ambient noise field as a forward model for fast computations of bottom loss data for a layered seabed. This model considers the effect of the array's finite aperture in the estimation of bottom loss and is extended to include the wind speed as the driving mechanism for the ambient noise field. The strength of this field relative to other unwanted noise mechanisms defines a signal-to-noise ratio, which is included in the inversion as a frequency-dependent parameter. The wind speed is found to have a strong impact on the resolution of seabed geoacoustic parameters as quantified by marginal probability distributions from Bayesian inversion of simulated data. The inversion method is also applied to experimental data collected at a moored vertical array during the MAPEX 2000 experiment, and the results are compared to those from previous active-source inversions and to core measurements at a nearby site. (C) 2012 Acoustical Society of America.
\end{abstract}

[http://dx.doi.org/10.1121/1.3688482]

PACS number(s): 43.30.Pc, 43.30.Ma, 43.60.Pt [AIT]

Pages: $2658-2667$

\section{INTRODUCTION}

Knowledge of seabed parameters such as sound speed, density, attenuation, and the sub-bottom layering structure is of primary importance for the development and validation of ocean acoustic models and for improvement of the performance of sonar systems. Among several techniques for remote sensing of the seabed, the use of wind-driven ambient noise recorded at a vertical line array (VLA) has been proposed ${ }^{1}$ as a convenient method with potential advantages over active acoustic and direct (core sampling) methods, such as less restrictive hardware, simpler deployment procedures, and minimal environmental impact. ${ }^{2}$ To the present, inversion of experimental ambient noise data has been approached by heuristic methods ${ }^{1}$ (i.e., manual search in the parameter space) and optimization procedures such as genetic algorithms, ${ }^{3}$ and studies of sensitivity of the noise field to environmental and array effects are available. ${ }^{4}$ Although the Bayesian approach for inversion of ambient noise has been explored in the past with simulated data, ${ }^{5}$ the strength of the noise field was not considered in the forward model, and results with experimental data have not yet been published. Two goals are pursued in this paper: First, the Bayesian framework is used to assess the impact of the surface wind speed in the estimation of geoacoustic parameters

\footnotetext{
a) Author to whom correspondence should be addressed. Electronic mail: jorgeq@uvic.ca
}

and their corresponding uncertainties in a study with simulated data. Second, the application of the inversion framework is demonstrated with experimental data collected in a moored array, and the geoacoustic parameters obtained are compared to simulations and to published results from active remote sensing methods and cores in the same region. ${ }^{6}$

Models for the wind-driven surface ambient noise have been developed ${ }^{7,8}$ and implemented, ${ }^{9}$ and the dependence of the resulting noise field on seabed parameters and frequency has been demonstrated. ${ }^{10}$ It has been shown with simulated and experimental data that an estimate of the seabed power reflection coefficient can be computed from the ratio of upward to downward energy fluxes obtained by beamforming ambient noise measured at a VLA. ${ }^{1,3}$ The resulting estimate resembles the power plane-wave reflection coefficient, smeared in angle due to the effect of the array's finite aperture. ${ }^{1}$ Alternatively, processing of the coherent noise field can produce an image of the seabed layering structure, ${ }^{2}$ the so called "passive fathometer," with results that have been confirmed by comparison to active surveys. ${ }^{11}$ Most of the work in processing ambient noise to extract layering structure has been devoted to adaptive beamforming techniques with the goal of improving the power to resolve fine layers. $^{2,11}$ In this paper, similar results are obtained by adopting a forward model based on conventional beamforming that represents the seabed as a series of fluid sediment layers. The proposed forward model considers the impact of signal-to-noise ratio (SNR), which is shown to have a strong 
effect on the uncertainties associated with the geoacoustic parameters.

Due to the non-linearity and large dimensionality of the problem, a numerical Bayesian framework is adopted in this work; a similar approach has been applied successfully to matched-field inversion ${ }^{12-14}$ and the inversion of reflection coefficients $^{6,15}$ measured using an active source, among others. The Bayesian method samples the joint posterior probability density (PPD) function of all model parameters to provide parameter estimates and uncertainties. In this work, simulated annealing ${ }^{16-18}$ with an exponential cooling schedule is used to determine the maximum a posteriori (MAP) model. Metropolis-Hastings sampling (MHS) ${ }^{19-21}$ is applied to determine marginal probability densities. In both cases, perturbations are applied in a principal-component parameter space, which is a rotated representation of the physical parameter space in which the axes align with the dominant correlation directions. ${ }^{21,22}$ This rotation provides a more efficient exploration of the parameter space and is particularly effective when strong correlations between parameters are present.

This paper is organized as follows: Sec. II describes the mathematical model for the ambient noise field. Section III summarizes the Bayesian framework for geoacoustic inversion. Section IV A investigates the effect of SNR on the ability to resolve seabed geoacoustic structure using simulated data. Section IV B applies the Bayesian inversion to ambient noise data measured in the MAPEX 2000 experiment, and the results are compared to those obtained by active-source methods. Section V presents conclusions.

\section{FORWARD MODEL}

In this section, a forward model for the computation of seabed bottom loss (BL) is described, and the results are compared to beamforming numerical simulations of the noise field from OASN, the ambient noise module from the wavenumber-integration model OASES. ${ }^{9}$

The quality of seabed reflection data derived from ambient noise is strongly affected by the wind speed. The magnitude of the surface wind ultimately determines the signal level available to probe the seabed, which must compete with isotropic electronic noise at the sensors as well as other sources of additive errors, allowing the definition of a frequency dependent SNR. The estimated reflection coefficients also contain artifacts introduced by beamforming, which smears the plane-wave reflection coefficient over angle. To include these distortion mechanisms in a forward model, the ray representation of the noise covariance developed by Harrison ${ }^{8}$ is adopted in this section and extended to include the SNR as an unknown parameter to be estimated.

This ray-tracing model for the covariance defines $c_{s}$ and $c_{b}$ as the sound speed at the surface and bottom of the water column, respectively, while $c_{r}$ is the mean sound speed along the $N$ elements of the VLA. When the VLA is located at a depth of relatively constant sound speed, the noise covariance matrix between elements $a$ and $b$ for rays arriving at angle $\theta^{\prime}$ (measured with respect to the horizontal) can be approximated as

$$
\begin{aligned}
S_{a b}(f) & =2 \pi \int_{-\pi / 2}^{\pi / 2} Q(f) A\left(\theta^{\prime}, f\right) e^{i k \Delta(a-b) \sin \theta^{\prime}} \cos \theta^{\prime} d \theta^{\prime} \\
1 & <a, b<N
\end{aligned}
$$

where $\Delta$ is the distance between elements, $k=2 \pi f / c_{r}$ is the wavenumber, $f$ is the frequency in Hertz, and $Q(f)$ is the strength of the dipoles at the air-water interface due to the surface wind. The amplitude term is defined as

$A\left(\theta^{\prime}, f\right)= \begin{cases}\frac{\sin \theta_{s}}{1-\left|R\left(\theta_{b}, f\right)\right|^{2}\left|R_{s}\left(\theta_{s}, f\right)\right|^{2}} & \text { if } 0<\theta^{\prime}<\pi / 2 \\ \frac{\sin \theta_{s}\left|R\left(\theta_{b}, f\right)\right|^{2}}{1-\left|R\left(\theta_{b}, f\right)\right|^{2}\left|R_{s}\left(\theta_{s}, f\right)\right|^{2}} & \text { if }-\pi / 2<\theta^{\prime}<0,\end{cases}$

where $\left|R\left(\theta_{b}, f\right)\right|^{2}$ and $\left|R_{s}\left(\theta_{s}, f\right)\right|^{2}$ are the power plane-wave reflection coefficients at the bottom and at the surface, respectively. The surface and bottom angles are related to $\theta^{\prime}$ by Snell's law as $\theta_{s}=\cos ^{-1}\left(\cos \left(\theta^{\prime}\right) c_{s} / c_{r}\right)$ and $\theta_{b}=\cos ^{-1}\left(\cos \left(\theta^{\prime}\right) c_{b} / c_{r}\right)$, and $A\left(\theta^{\prime}, f\right)$ can be obtained from the plane-wave reflection coefficient for a stack of $L$ layers. ${ }^{23}$ Because $c_{s} \approx 1524 \mathrm{~m} / \mathrm{s}$ and $c_{r} \approx c_{b} \approx 1512 \mathrm{~m} / \mathrm{s}$, there are rays connecting the surface and the bottom at all angles $0^{\circ} \leq \theta_{b} \leq 90^{\circ}$, which might not be the case for environments with more complicated sound speed profiles. ${ }^{1}$ Notice that Eqs. (1) and (2) are the same as Eq. (8) from Harrison ${ }^{8}$ with the terms $m=1, \gamma=90^{\circ}$ and assuming zero volume absorption. In addition, throughout this paper $\left|R_{s}\left(\theta_{s}, f\right)\right|^{2}$ $=1$. These assumptions are briefly discussed in the following text.

By defining the array steering vector $\mathbf{v}(\theta, f)=[1$, $\left.e^{-i k \Delta \sin \theta}, \ldots, e^{-i k(N-1) \Delta \sin \theta}\right]^{H}$ (where $H$ indicates Hermitian transpose), the array response in direction $\theta$ is

$$
\begin{aligned}
& Y(\theta, f)=\frac{1}{N^{2}} \mathbf{v}^{H}(\theta, f) \mathbf{S}(f) \mathbf{v}(\theta, f), \\
& \mathbf{S}(f)=\left(\begin{array}{cccc}
s_{11}(f) & s_{12}(f) & \cdots & s_{N N}(f) \\
s_{21}(f) & s_{22}(f) & \cdots & s_{2 N}(f) \\
\vdots & \vdots & \cdots & \vdots \\
s_{N 1}(f) & s_{N 2}(f) & \cdots & s_{N N}(f)
\end{array}\right) .
\end{aligned}
$$

With the change of variables $x^{\prime}=\sin \theta^{\prime}$ in Eq. (1) and $x=\sin \theta$ in Eq. (3), the product $\mathbf{v}^{H}(\theta, f) \mathbf{S}(f)$ is a row vector of the form

$$
\begin{aligned}
& \mathbf{v}^{H}(\theta, f) \mathbf{S}(f)= \\
& 2 \pi Q(f)\left(\begin{array}{c}
\int_{-1}^{1} A\left(x^{\prime}, f\right) \sum_{n=0}^{N-1} e^{-i k n \Delta x^{\prime}} e^{i k n \Delta x} d x^{\prime} \\
\int_{-1}^{1} A\left(x^{\prime}, f\right) \sum_{n=0}^{N-1} e^{-i k n \Delta x} e^{i k(n-1) \Delta x^{\prime}} d x^{\prime} \\
\vdots \\
\int_{-1}^{1} A\left(x^{\prime}, f\right) \sum_{n=0}^{N-1} e^{-i k n \Delta x} e^{i k(n-N+1) \Delta x^{\prime}} d x^{\prime}
\end{array}\right)^{T},
\end{aligned}
$$




$$
\begin{aligned}
Y(\theta, f)= & \frac{2 \pi}{N^{2}} Q(f) \int_{-1}^{1} A\left(x^{\prime}, f\right) \\
& {\left[\sum_{q=0}^{N-1} e^{i k q \Delta x}\left(\sum_{n=0}^{N-1} e^{-i k n \Delta x} e^{i k(n-q) \Delta x^{\prime}}\right)\right] d x^{\prime} . }
\end{aligned}
$$

Notice that the quantity in square brackets is $\mid\left[\left.\sum_{n=0}^{N-1} e^{-i k n \Delta\left(x-x^{\prime}\right)}\right|^{2}\right.$ and that the limits of the integral can be extended to $\pm \infty\left(\right.$ since $A\left(x^{\prime}, f\right)=0$ for $\left.\left|x^{\prime}\right|>1\right)$. Then the array response is

$$
Y(\theta, f)=\frac{2 \pi}{N^{2}} Q(f) A(x, f) *\left|\sum_{n=0}^{N-1} e^{-i k n \Delta x}\right|^{2} .
$$

where $*$ is the convolution operator, yielding a forward model that can be implemented efficiently using the fast Fourier transform to compute the convolution.

In addition to the acoustic field established by the surface wind (i.e., the signal in this paper), isotropic white noise with power $\sigma_{w}^{2}$ is also detected at the elements of the array. The sensor noise contributes a diagonal term to the covariance matrix, and the estimated $\mathrm{BL}$ in decibels is then

$$
\begin{aligned}
& \mathrm{BL}(\theta, f)=-10 \log \left|\frac{Y(\theta, f) N+Q(f) 10^{-\mathrm{SNR}_{f / 10}}}{Y(-\theta, f) N+Q(f) 10^{-\mathrm{SNR}_{f / 10}}}\right|, \\
& 0<\theta<\pi / 2,
\end{aligned}
$$

where $\mathrm{SNR}_{f}=10 \log _{10}\left[Q(f) / \sigma_{w}^{2}\right]$ is the SNR in decibels. The BL evaluated at $V$ angles and $F$ frequencies is collected into a single vector as

$$
\mathbf{d}_{L}(\mathbf{m})=\left(\begin{array}{c}
\operatorname{BL}\left(\theta_{1}, f_{1}\right) \\
\operatorname{BL}\left(\theta_{2}, f_{1}\right) \\
\vdots \\
\operatorname{BL}\left(\theta_{V}, f_{1}\right) \\
\operatorname{BL}\left(\theta_{1}, f_{2}\right) \\
\vdots \\
\operatorname{BL}\left(\theta_{V}, f_{F}\right)
\end{array}\right) .
$$

In Eq. (8), the subscript $L$ indicates that the BL has been computed for a seabed consisting of $L$ layers (including an under-lying half space) with the $l$ th layer characterized by the sound speed $c_{l}$, density $\rho_{l}$, attenuation $\alpha_{l}$, and thickness $h_{l}$ (the $L$ th layer is semi-infinite). These parameters are arranged in the vector $\mathbf{m}$ as described in the following text in Sec. III.

To test the accuracy of the forward model, simulated data were generated using OASES at frequencies of 635, 800, 1008, 1270 , and $1400 \mathrm{~Hz}$ with SNRs of $-7.8,-7.1,-6.4,-5.6$, and $-5.2 \mathrm{~dB}$, respectively. The sediment for this simulation consists of three layers with geoacoustic parameters given in Table I. This environment was chosen to match the profile estimated from the experimental data presented in Sec. IV B.

Figure 1(a) shows the sound-speed profile of the water column (used throughout this paper) obtained from a CTD
TABLE I. Parameters for the three-layer model used to generate simulated data.

\begin{tabular}{lcccc}
\hline \hline & \multicolumn{4}{c}{ Parameters } \\
\cline { 2 - 5 } & $\begin{array}{c}c_{l} \\
\text { Layer }\end{array}$ & $\begin{array}{c}\rho_{l} \\
(\mathrm{mg} / \mathrm{s})\end{array}$ & $\begin{array}{c}\alpha_{l} \\
(\mathrm{~dB} / \lambda)\end{array}$ & $\begin{array}{c}h_{l} \\
(\mathrm{~m})\end{array}$ \\
\hline 1 & 1520 & 1542 & 0.2 & 0.8 \\
2 & 1750 & 2306 & 1.5 & 0.8 \\
3 & 1598 & 1813 & 0.4 & $\infty$ \\
\hline \hline
\end{tabular}

measurement taken at the VLA during the MAPEX 2000 experiment. ${ }^{1}$ Figure 1(b) shows the percent error between the BL estimated by beamforming of simulated ambient noise data from OASES and from the forward model described in the preceding text, at the five frequencies and angles from $13^{\circ}$ to $89^{\circ}$ spaced every $4^{\circ}$ (i.e., $F=5$ and $V=20$ ). In all cases, the model in Eq. (8) is consistent with the numeric solution from OASES, giving percent errors smaller than $2.6 \%$ and generally below $1 \%$.

Because the SNRs are not known when considering measured ambient noise, they are treated as unknown parameters in the Bayesian inversion. The distortion introduced by this term is accentuated at angles close to normal incidence, where the white noise tends to fill in regions of small reflection coefficient (high BL).

As mentioned before, some assumptions have been made in the model to reduce the number of parameters in the search space. First, although the $\sin \theta_{s}$ term in Eq. (2) can be written more generally ${ }^{8}$ as $\sin ^{2 m-1} \theta_{s}$, one of the strengths of the approach in Eq. (7) is that it is relatively immune to the noise directionality. ${ }^{1}$ To support this statement, a comparison between OASN-generated noise with $m=2$ and the forward model with $m=1$ (not shown) revealed typical differences of less than $4 \%$.

A weak dependence of Eq. (7) to the surface power plane-wave reflection coefficient was also found in preliminary simulations. To give a numerical example, the Schulkin-Marsh model ${ }^{24}$ can be used to obtain reasonable bounds for the amount of power loss due to rough surface scattering. For wind speeds around 22 knots (kts), wave (a)

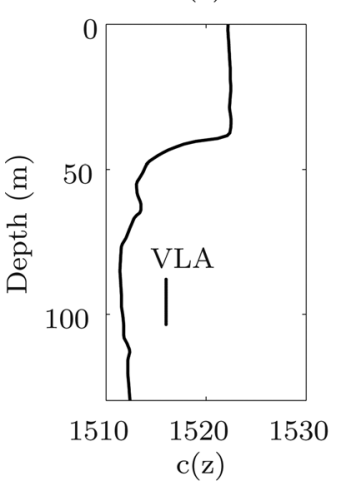

(b)

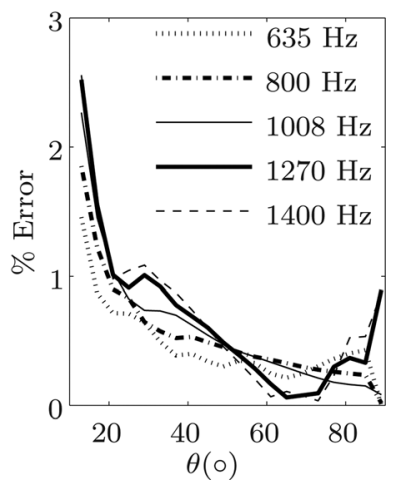

FIG. 1. (a) Sound-speed profile in the water column measured during the MAPEX 2000 experiment ${ }^{1}$ with depth extent of the VLA indicated. (b) Percent error for the BL estimated from simulated data generated by OASES and by the forward model, using the parameters in Table I. 
heights of $2.5 \mathrm{~m}$ have been suggested. ${ }^{24}$ For the frequency range in this paper, losses from 3.7 to $5.5 \mathrm{~dB} /$ bounce can be expected. This would introduce errors of $1 \%$ when estimating the bottom loss in Eq. (7), which is unlikely to significantly affect the inversion.

Regarding volume attenuation, the impact of water absorption at frequencies of $10 \mathrm{kHz}$ has been quantified as a function of array depth. ${ }^{4}$ It was observed that for an array below the middle of the water column, the water absorption introduces artifacts in the bottom loss on the order of $1.5 \mathrm{~dB}$ at most and typically less than $1 \mathrm{~dB}$. Furthermore, the effect of water absorption is mostly evident at low grazing angles (i.e. $\theta<20^{\circ}$ ), which in this paper have been excluded. At the lower frequencies in this work, water absorption is not likely to have a significant impact on the inversions.

Section IV shows that low values of SNR limit the power to resolve seabed parameters.

\section{BAYESIAN INVERSION}

This section summarizes the Bayesian inversion approach following Dosso and Dettmer. ${ }^{14}$ For notational convenience, all the parameters are collected into a single vector $\mathbf{m}$ as

$$
\mathbf{m}=\left[c_{1} \rho_{1} \alpha_{1} h_{1} \ldots c_{L} \rho_{L} \alpha_{L} \mathrm{SNR}_{1} \mathrm{SNR}_{2} \ldots \mathrm{SNR}_{F}\right]^{T} .
$$

The vector of data $\mathbf{d}$ input to the Bayesian inversion has the same form as Eq. (8) except that the predicted (modeled) $\operatorname{BL}(\theta, f)$ is replaced by measured $\overline{\mathrm{BL}}(\theta, f)$, defined as

$$
\begin{gathered}
\overline{\mathrm{BL}}(\theta, f)=-10 \log _{10}\left|\frac{\mathbf{v}^{T}(\theta, f) \mathbf{D}(f) \mathbf{v}(\theta, f)}{\mathbf{v}^{T}(-\theta, f) \mathbf{D}(f) \mathbf{v}(-\theta, f)}\right|, \\
0<\theta<\pi / 2
\end{gathered}
$$

where $\mathbf{D}(f)$ is the ambient-noise covariance matrix, estimated by averaging over snapshots of the ambient noise in the frequency domain, following Siderius et al. ${ }^{11}$ The difference between the measured and the predicted data defines the residuals $\mathbf{r}=\mathbf{d}-\mathbf{d}_{L}(\mathbf{m})$, which are here assumed to be zeromean Gaussian-distributed with data covariance matrix $\mathbf{C}_{d}$. The PPD is given by

$$
P(\mathbf{m} \mid \mathbf{d}, L)=\frac{P(\mathbf{d} \mid \mathbf{m}, L) P(\mathbf{m} \mid L)}{P(\mathbf{d} \mid L)},
$$

where $P(\mathbf{d} \mid \mathbf{m}, L)$ is the likelihood function, $P(\mathbf{m} \mid L)$ is the prior information (described in Sec. IV), and $P(\mathbf{d} \mid L)$ is a normalizing constant. Under the assumption of Gaussian- distributed residuals, the likelihood can be written as

$$
P(\mathbf{d} \mid \mathbf{m}, L)=\frac{1}{(2 \pi)^{N / 2}\left|\mathbf{C}_{d}\right|^{1 / 2}} \exp \left(-\frac{1}{2} \mathbf{r}^{\mathrm{T}} \mathbf{C}_{\mathrm{d}}^{-1} \mathbf{r}\right) .
$$

For the experimental data in this paper, $\mathbf{C}_{d}$ was estimated from the autocovariance function of residuals from a preliminary inversion by the iterative procedure described in Dosso et al. ${ }^{25}$ Once $\mathbf{C}_{d}$ is estimated, the PPD can be calculated. Because analytical solutions for the PPD are gen- erally not available for non-linear problems, Markov chain Monte Carlo (MCMC) methods can be used to sample from this distribution. Typical PPD properties that are considered include marginal probability distributions $P\left(m_{l} \mid \mathbf{d}, L\right)$ and the model covariance matrix $\mathbf{C}_{\mathbf{m}}$, which are computed as

$$
\begin{aligned}
& P\left(m_{l} \mid \mathbf{d}, L\right)=\int \delta\left(m_{l}-m_{l}^{\prime}\right) P\left(\mathbf{m}^{\prime} \mid \mathrm{d}, L\right) d \mathbf{m}^{\prime} \\
& \mathbf{C}_{\mathrm{m}}=\int\left(\mathbf{m}^{\prime}-<\mathbf{m}>\right)\left(\mathbf{m}^{\prime}-<\mathbf{m}>\right)^{T} P\left(\mathbf{m}^{\prime} \mid \mathbf{d}, L\right) d \mathbf{m}^{\prime} \\
& <\mathbf{m}>=\int \mathbf{m}^{\prime} P\left(\mathbf{m}^{\prime} \mid \mathbf{d}, L\right) d \mathbf{m}^{\prime}
\end{aligned}
$$

while correlation coefficients are found by normalizing the elements of the covariance matrix as $R_{i j}=\mathbf{C}_{\mathrm{m} i j} / \sqrt{\mathbf{C}_{\mathrm{m} i i} \mathbf{C}_{\mathrm{m} j j}}$.

In this paper, the MAP model $\mathbf{m}_{\text {MAP }}$ was estimated by maximizing Eq. (11) using simulated annealing ${ }^{16-18}$ with principal-component rotation and an exponential cooling schedule $T_{j}=T_{0}\left(100 T_{0}\right)^{-j / J}$, where $T_{0}$ is the initial temperature set to $T_{0}=10 \mathrm{VF}, j$ is the current temperature step, and $J$ is the total number of steps. PPD properties in Eqs. (13) to (15) were estimated by applying MHS, using a principalcomponent rotation and Cauchy proposal distributions scaled by the square root of the principal-component variances to increase efficiency. ${ }^{21}$

Model selection is carried out in this paper by minimizing the BIC defined as ${ }^{26}$

$$
\operatorname{BIC}(L)=-2 \log _{e}\left[P\left(\mathbf{m}_{\mathrm{MAP}} \mid \mathbf{d}, L\right)\right]+M \log _{e}(N),
$$

where $M=4 L-1+F$ is the total number of model parameters and $N=V F$ is the total number of data.

\section{INVERSION OF AMBIENT NOISE DATA}

In this section, the effect of the surface wind speed in geoacoustic parameter resolution is studied using Bayesian inversion of simulated ambient noise data. Following this, Bayesian inversion is applied to experimental data collected at a moored VLA (Sec. IV B). The common features between these inversions are described first here.

The prior information $P(\mathbf{m} \mid L)$ used in all inversions consists of uniform distributions with bounds $c_{l} \in[1450$, $1950] \mathrm{m} / \mathrm{s}, \rho_{l} \in[1350,2400] \mathrm{kg} / \mathrm{m}^{3}, \alpha_{l} \in[0,1.7] \mathrm{dB} / \lambda$, and $h_{l} \in[0.1,1.5] \mathrm{m}, \forall l \leq L$. Due to the availability of a core sample near the location of the array, the sound speed limits for the top layer were set to $c_{1} \in[1450,1600] \mathrm{m} / \mathrm{s}$. In addition, a mutual constraint among density, attenuation, and sound speed was imposed based on a collection of laboratory measurements from sediments of different grain size (see Figs. 5.2 and 5.9 from Jackson et al. ${ }^{27}$ ). This constraint limits the search domain to plausible solutions in which sediments with higher densities are likely to exhibit higher attenuations and sound speeds. Given a density $\rho_{l}$, the sound speed and attenuation are bounded between $\left[c_{l}^{-}, c_{l}^{+}\right]$and $\left[\alpha_{l}^{-}, \alpha_{l}^{+}\right]$defined as 


$$
\begin{aligned}
c_{l}^{+} & =1529.4\left(1.7-0.98 \frac{\rho_{l}}{1000}+0.38\left(\frac{\rho_{l}}{1000}\right)^{2}\right), \\
c_{l}^{-} & =1529.4\left(1.6-0.98 \frac{\rho_{l}}{1000}+0.34\left(\frac{\rho_{l}}{1000}\right)^{2}\right), \\
\alpha_{l}^{+} & =1.5294\left(0.00332 e^{0.00275 \rho_{l}}+0.1\right), \\
\alpha_{l}^{-} & =0 .
\end{aligned}
$$

\section{A. Geoacoustic resolution as a function of wind speed}

Simulated ambient noise data were generated from Eq. (8) using the environment described in Table I and the soundspeed profile in Fig. 1(a). The frequency-dependent source strength was computed according to Ingenito et al. ${ }^{10}$ as $Q(f)=10^{\left[44.58+(0.14) f^{0.37} V s\right] / 10}$, where $V_{s}$ is the surface wind speed in knots. Wind speeds of 5, 10, and 15 kts are considered here.

Similar to the experiment described in Sec. IV B, the water column is $130-\mathrm{m}$ deep and the receiving array has 32 elements spaced by $0.5 \mathrm{~m}$ from $88-104 \mathrm{~m}$ depth. The level of isotropic white noise [relative to $Q(f)$ ] at the array is fixed to $10 \log _{10} \sigma_{w}^{2}=60 \mathrm{~dB}$, and uncorrelated Gaussian errors with $\sigma_{d}^{2}=0.2$ were added to the simulated data. For all wind speeds, the same realization of Gaussian errors was added to the simulated data, and therefore the misfit at the true model is the same in all cases. The goal of these simulations is to study the effect of the wind speed and the SNR on the ability to resolve geoacoustic parameters.

Simulated datasets are presented in Fig. 2. Each curve corresponds to data at one of the three wind speeds and five frequencies selected for inversion, with grazing angles from $13^{\circ}$ to $89^{\circ}$ at each frequency. The corresponding predicted data computed for the MAP models obtained from the simulated annealing optimization are plotted as solid lines. In all cases, the model fits the data, yielding statistically independent residuals of variance approximately $\sigma_{d}^{2}$ in agreement
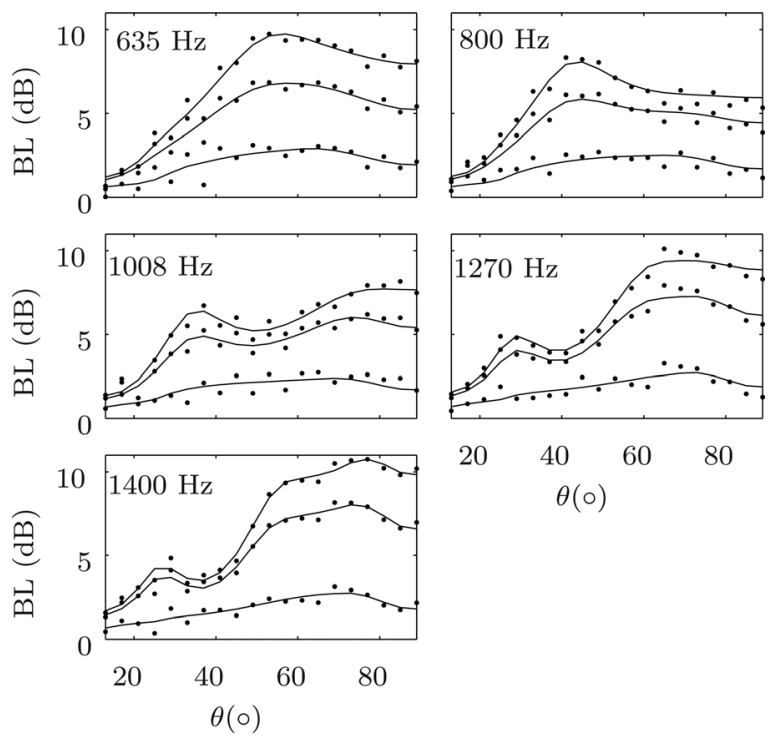

FIG. 2. Simulated data (dots) and data predicted for the MAP model (solid lines) for wind speeds of 5, 10, and $15 \mathrm{kts}$ (bottom, middle, and top curve in each panel, respectively). with the uncorrelated Gaussian errors added to the simulated data. Differences in the resulting MAP models and sediment profiles can be thought as caused by a reduction in the information content of the data regarding the geoacoustic parameters as the wind speed decreases.

The variation in information content with wind speed can be observed from the marginal probability profiles in Fig. 3, obtained from MCMC sampling. The parameters of the top layer are in good agreement with the true model even in the worst case of low wind speed [Fig. 3(c)]. Deeper layers are still resolved for wind speeds of 15 and $10 \mathrm{kts}$ for
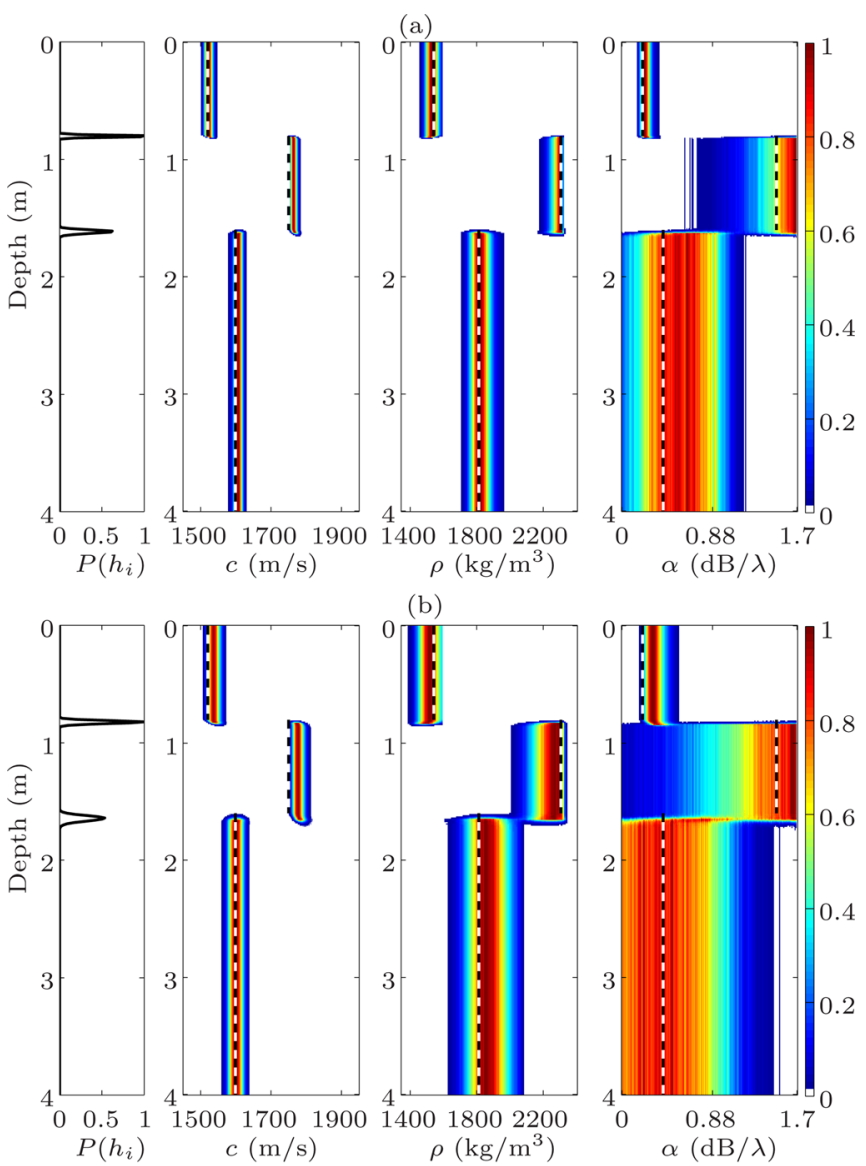

(b)
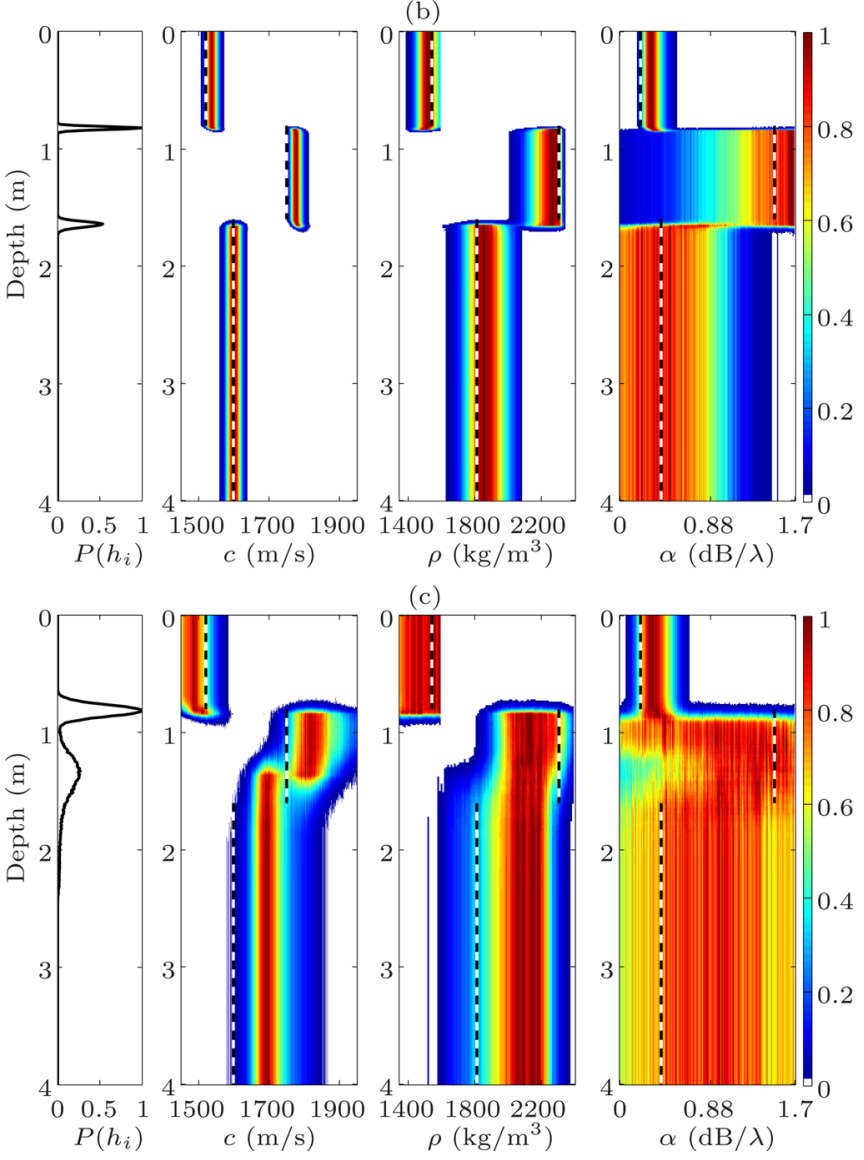

FIG. 3. (Color online) Marginal probability profiles for the layer thickness, sound speed, density, and attenuation obtained from simulated data with (a) $V_{s}=15 \mathrm{kts}$, (b) $V_{s}=10 \mathrm{kts}$, and (c) $V_{s}=5 \mathrm{kts}$. Dashed lines indicate the true model from Table I. The profiles are normalized to have a maximum of 1 ; profiles for $c, \rho$ and $\alpha$ are normalized independently at each depth. 

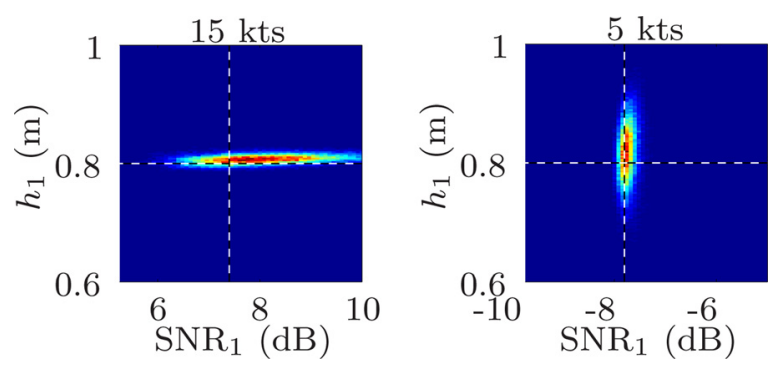

FIG. 4. (Color online) Joint marginal PPDs of $h_{1}$ vs $\mathrm{SNR}_{1}$ for wind speeds of $V_{s}=15$ and $V_{s}=5 \mathrm{kts}$. Dashed lines indicate true values.

which the SNR is greater than $0 \mathrm{~dB}$ at almost all frequencies. However, at $5 \mathrm{kts}$, all SNRs are lower than $-5 \mathrm{~dB}$, resulting in loss of geoacoustic resolution for all parameters. In Figs. 3(a) and 3(b), the marginal PPDs for sound speeds and thicknesses are compact, indicating good geoacoustic resolution. On the other hand, the support of the PPDs for densities and attenuations tends to fill a greater proportion of their prior distributions. This behavior can be explained from the frequency-angle structure of the bottom loss, in which the ratio between layer thickness and sound speed determines the location of periodic fringes. The attenuation and the density remain as parameters that mostly influence the energy level, which is a weaker feature of the bottom loss.

Considering joint marginal PPDs illustrates the correlations between parameters. Figure 4 shows joint marginal PPDs for SNR and layer thickness for wind speeds of $V_{s}=15$ and $5 \mathrm{kts}$. The trade off between sediment thickness and SNR resolution is observed: At high SNR ( $\left.V_{s}=15 \mathrm{kts}\right)$, the estimated bottom loss in Eq. (7) is dominated by $Y_{s}(\theta)$, giving a narrow range of potential solutions for $h_{1}$, which is constrained by the angle-frequency structure of the bottom loss. At low SNR ( $V_{s}=5 \mathrm{kts}$ ), the information in the data is dominated by the frequency-dependent SNR, resulting in tight uncertainties for the estimated SNR and low resolution for the geoacoustic parameters. Other marginal PPDs are shown in Sec. IV B and compared to those obtained from experimental data.

\section{B. Experimental data}

Ambient noise data were collected on November 22, 2000, during the MAPEX 2000 experiment, ${ }^{11}$ carried out on the Malta Plateau using a moored VLA located at (36.44357 N lat., 14.77618 E long.). During the experiment, ambient noise was recorded at a sampling rate of $6000 \mathrm{~Hz}$ with an 80-element array consisting of three sub-arrays. The data used in this paper correspond to the middle subarray with 32 equally spaced elements spanning 88-104 m depth. The sound-speed profile is shown in Fig. 1(a), and the wind speed was measured to be 20-22 kts.

Pre-processing of the experimental data to obtain BL was carried out using conventional beamforming of the covariance matrix, estimated from $7 \mathrm{~min}$ of recorded data by averaging over snapshots in the frequency domain. Each snapshot was computed from $1.4 \mathrm{~s}$ of data, following Siderius et al. ${ }^{11}$

To determine the optimal seabed parametrization, a BIC study $^{26}$ which considered from two to five layers was carried (a)

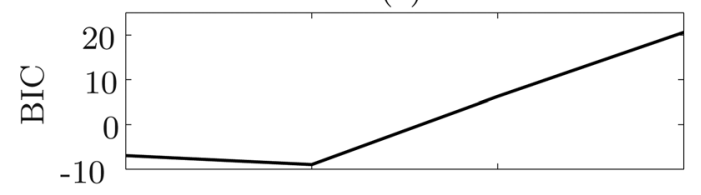

(b)

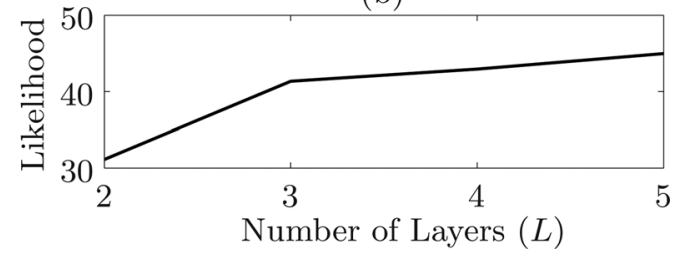

FIG. 5. (a) BIC for the experimental data as a function of the number of layers in the forward model; (b) corresponding likelihood function.

out by evaluating Eq. (16). Figure 5 shows that the BIC has a minimum at three layers (including the halfspace), indicating that this is the optimal parameterization. Figure 6 shows the fit to the experimental data for the three-layer MAP model. For the inversion results shown here, the errors between the measured and modeled data are assumed to be Gaussian distributed with covariance matrices $\mathbf{C}_{d}$ at each frequency, estimated as mentioned in Sec. III. Figure 7 shows the estimated covariance matrices as well as the autocorrelation of the residuals. At each frequency, the covariance matrices correspond to correlated residuals with angledependent variances, indicated by the varying amplitude along the main diagonal. The autocorrelation of the raw residuals (dashed line) has a relatively wide center peak at most frequencies, indicating correlation between residuals. The autocorrelation for the standardized residuals (solid line) indicates improvement, producing narrower peaks and reducing the amplitude of the tails, which supports the use of the full estimated data covariance matrix in the inversion. In addition, the standardized residuals also pass the Kolmogorov-Smirnov (KS) test for Gaussianity and the runs
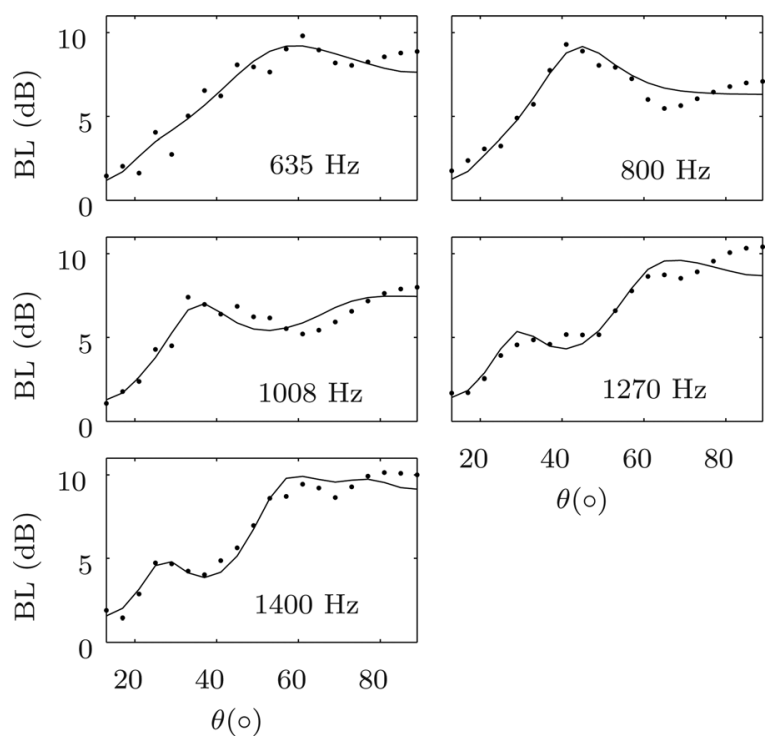

FIG. 6. Experimental BL (dots) compared to the predicted BL data evaluated at $\mathbf{m}_{\mathrm{MAP}}$ corresponding to the 3-layer model (solid lines). 


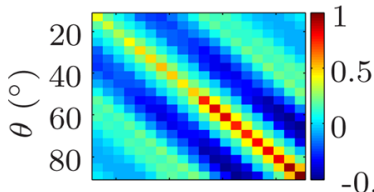

$20 \quad 40 \quad 60 \quad 80$

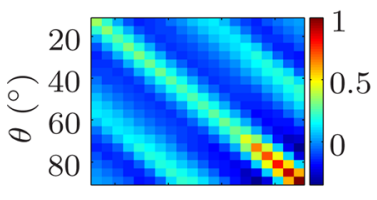

$20 \quad 40 \quad 60 \quad 80$

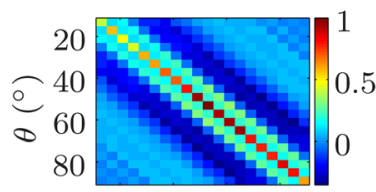

$20 \quad 40 \quad 60 \quad 80$

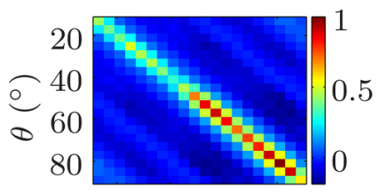

$20 \quad 40 \quad 60 \quad 80$
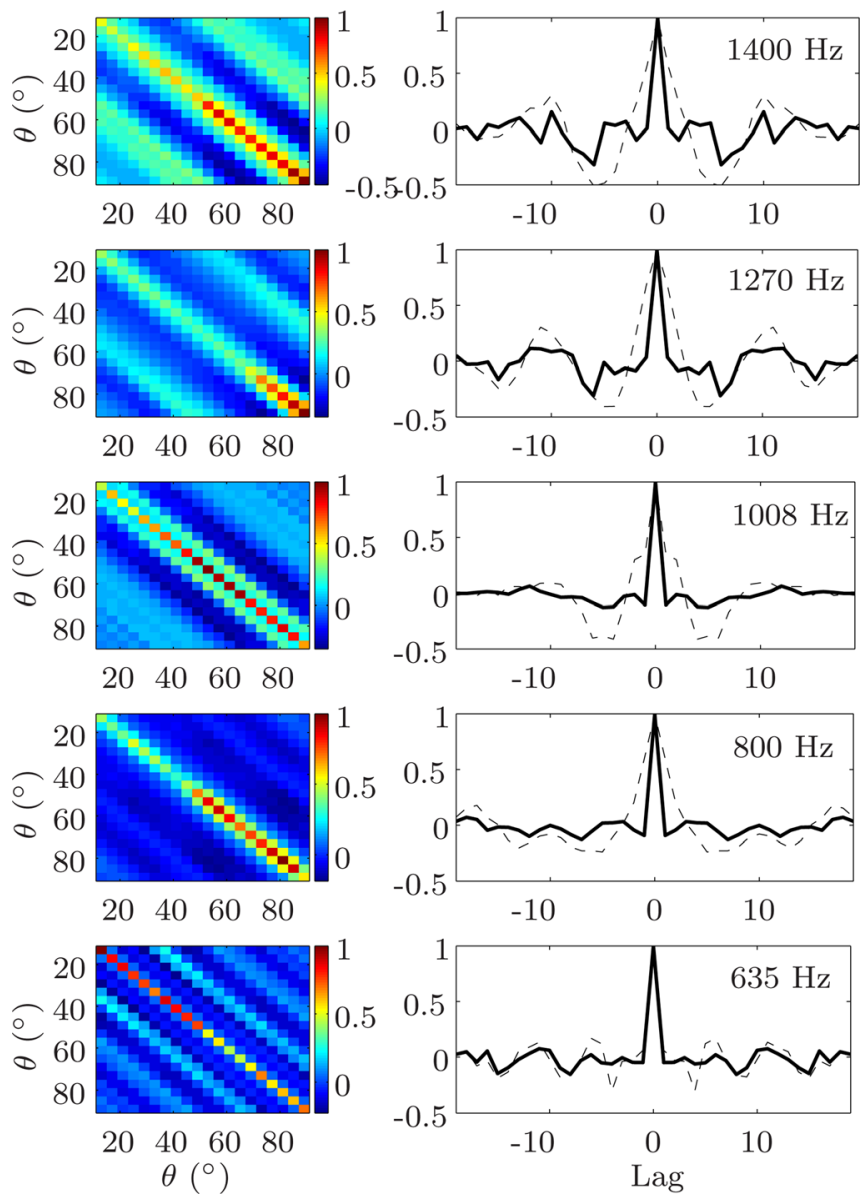

FIG. 7. (Color online) Estimated data covariance matrix $\mathbf{C}_{d}$ at each of the five frequencies and corresponding autocorrelation of the standardized (solid) and raw (dashed) residuals. Each plot is normalized to a maximum of 1.

test for randomness at a 0.05 level, with the $P$ values given in Table II. Thus there is no evidence against the initial assumption of Gaussian statistics for the residuals.

The marginal probability profiles from Bayesian inversion of the data are shown in Fig. 8(a). These PPDs resemble the results from the simulated inversions with $V_{s}=15 \mathrm{kts}$ and $V_{s}=10 \mathrm{kts}$ in Fig. 3: All geoacoustic parameters are well determined over the top layer, and then the distributions widen with depth particularly for density and attenuation. In Fig. 8(a), measurements of the sound speed and the density from core samples from a nearby area are plotted overlying the probability profile. The marginal PPDs for sound speed and density are in good agreement with the corresponding cores up to $0.9 \mathrm{~m}$ depth for the sound speed (total core depth) and to $1.5 \mathrm{~m}$ deep for the density. Beyond this depth, the estimated density decreases but the core measurement

TABLE II. Results of KS and runs tests for the standardized and the raw (in parenthesis) residuals.

\begin{tabular}{lccccc}
\hline \hline & \multicolumn{5}{c}{ Frequencies (Hz) } \\
\cline { 2 - 6 } & 635 & 800 & 1008 & 1270 & 1400 \\
\hline Runs & $1.0(1.0)$ & $1.0(0.1)$ & $0.9(0.9)$ & $0.1(0.01)$ & $0.1(0.1)$ \\
KS & $0.5(0.9)$ & $0.9(1.0)$ & $0.6(0.3)$ & $0.9(0.7)$ & $0.4(0.7)$ \\
\hline \hline
\end{tabular}
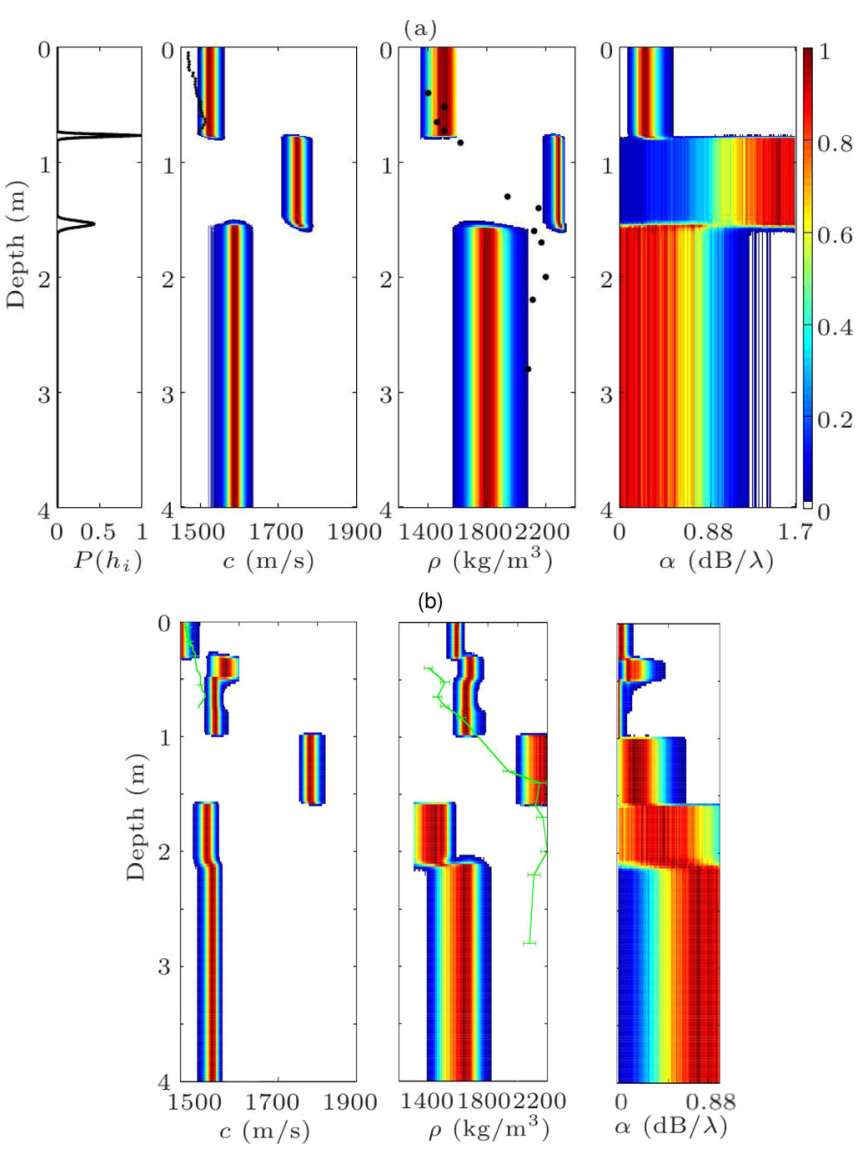

FIG. 8. (Color online) (a) Marginal probability profiles from the experimental bottom loss inversion. The dots are sound speed and density core measurements taken at a location $\sim 400 \mathrm{~m}$ away from the array; (b) similar profile from Dettmer et al. ${ }^{6}$ obtained by active-source methods with lines indicating the same core measurements as in (a). Note that the higher bounds for the density and the attenuation in (b) are $2200 \mathrm{~kg} / \mathrm{m}^{3}$ and $1 \mathrm{~dB} / \lambda$, respectively.

remains at a value $\sim 2100 \mathrm{~kg} / \mathrm{m}^{3}$. This disagreement in the density is also observed in the profile in Fig. 8(b), obtained by Bayesian inversion of active-source spherical reflection coefficient data (details of this active- source experiment can be found in Dettmer et al. ${ }^{6}$ ) This suggests a possible problem with the core sample (e.g., compaction of the lower part of the core) because both profiles were obtained from independent acoustic measurement methods.

The active measurements used to produce Fig. 8(b) were collected at eight frequencies ranging from 300 to $1600 \mathrm{~Hz}$ using a towed source and a single stationary receiver located at $(36.4441 \mathrm{~N}$ lat., $14.7804 \mathrm{E}$ long.), i.e., $\sim 400 \mathrm{~m}$ away from the moored array used in this study. Comparison of the "passive" and "active" PPDs in Fig. 8 reveals similarity in a general sense: There is a dominant top layer of thickness $\sim 0.9 \mathrm{~m}$ followed by a sharp increase in the density and sound speed down to $1.5 \mathrm{~m}$, and deeper layers with lower density and sound speed. The BIC for the active data indicated a model with six layers (including the bottom halfspace) rather than three layers as in the results in this paper. The upper-most sediments in the "passive" profile are represented by a single layer $\sim 0.8 \mathrm{~m}$ deep, as opposed to three sublayers in the "active" profile. This could be due to differences in the information content from the active data set compared to the ambient noise as well as geographic 
variability of the seabed structure. It is noted that the physical constraints between density, sound speed, and attenuation from Eq. (17) were not utilized in the inversion of active data, ${ }^{6}$ and this could explain the differences in the estimated sediment attenuation between 0.9 and $1.5 \mathrm{~m}$.

To further investigate the ambient-noise geoacoustic inversion results, Fig. 9 shows the measured active-source reflection coefficient data $^{6}$ (dots) compared to predicted data. To produce this figure, the sampled models illustrated in the marginal distributions from Fig. 8(a) were used to compute spherical reflection coefficients, which are presented as a mean (solid line) \pm 3 standard deviations (dashed lines) on top of the experimental active-source measurements. The proposed model from passive inversion fits the active dataset very well at low frequencies but starts to diverge at $635 \mathrm{~Hz}$. This could be due to requiring more resolution at higher frequencies, suggesting that the "passive" and "active" models are equivalent at a coarse scale level. In all cases, the fit is better at low grazing angles, although in almost all cases the predicted reflection coefficients follow a
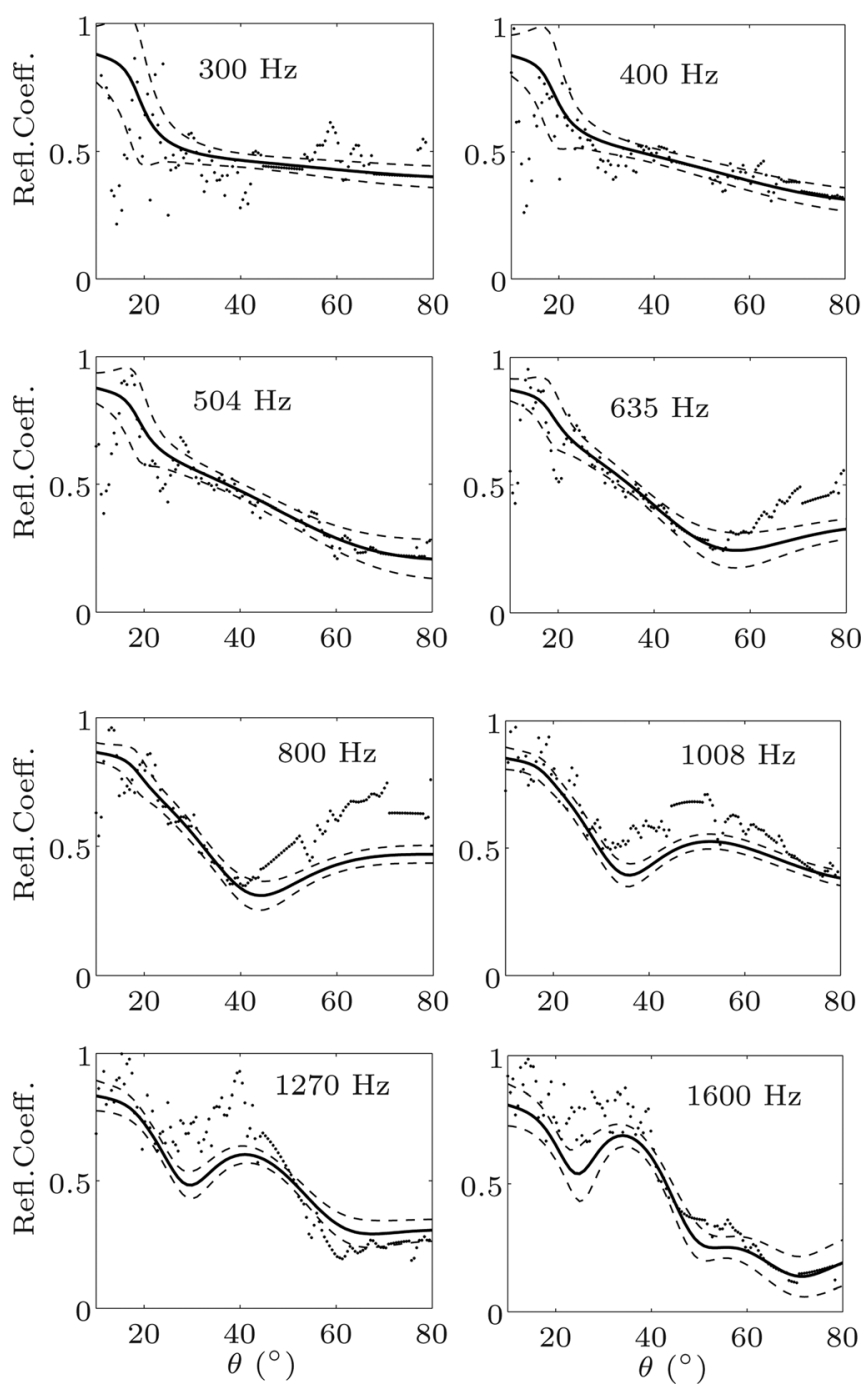

FIG. 9. Spherical reflection coefficients for the three-layer model from Fig. 8, compared to active-source measurements (dots) from Dettmer et al. ${ }^{6}$ The lines represent the mean reflection coefficient (solid line) \pm 3 standard deviations (dashed lines). similar trend of minima and maxima as the active-source data. The active data in Fig. 9 should not be taken as ground truth to judge the accuracy of the passive technique presented here, because other factors such as range-dependent variability of the sediment structure could play a fundamental role in this geographic area.

The importance of considering SNR in ambient noise inversion can be quantified by study of parameter correlations. Figure 10 shows the correlation coefficient matrices for the inversion of measured data and simulated data with $V_{s}=15 \mathrm{kts}$. In both cases, there is significant correlation between some of the SNR parameters at different frequencies and between SNR and layer thicknesses, sound speeds, and densities. For example, the pairs $\mathrm{SNR}_{1}$ vs $\rho_{1}$ and $\mathrm{SNR}_{1}$ vs $c_{1}$ in Fig. 10(a) exhibit correlations of -0.66 and 0.64 ,

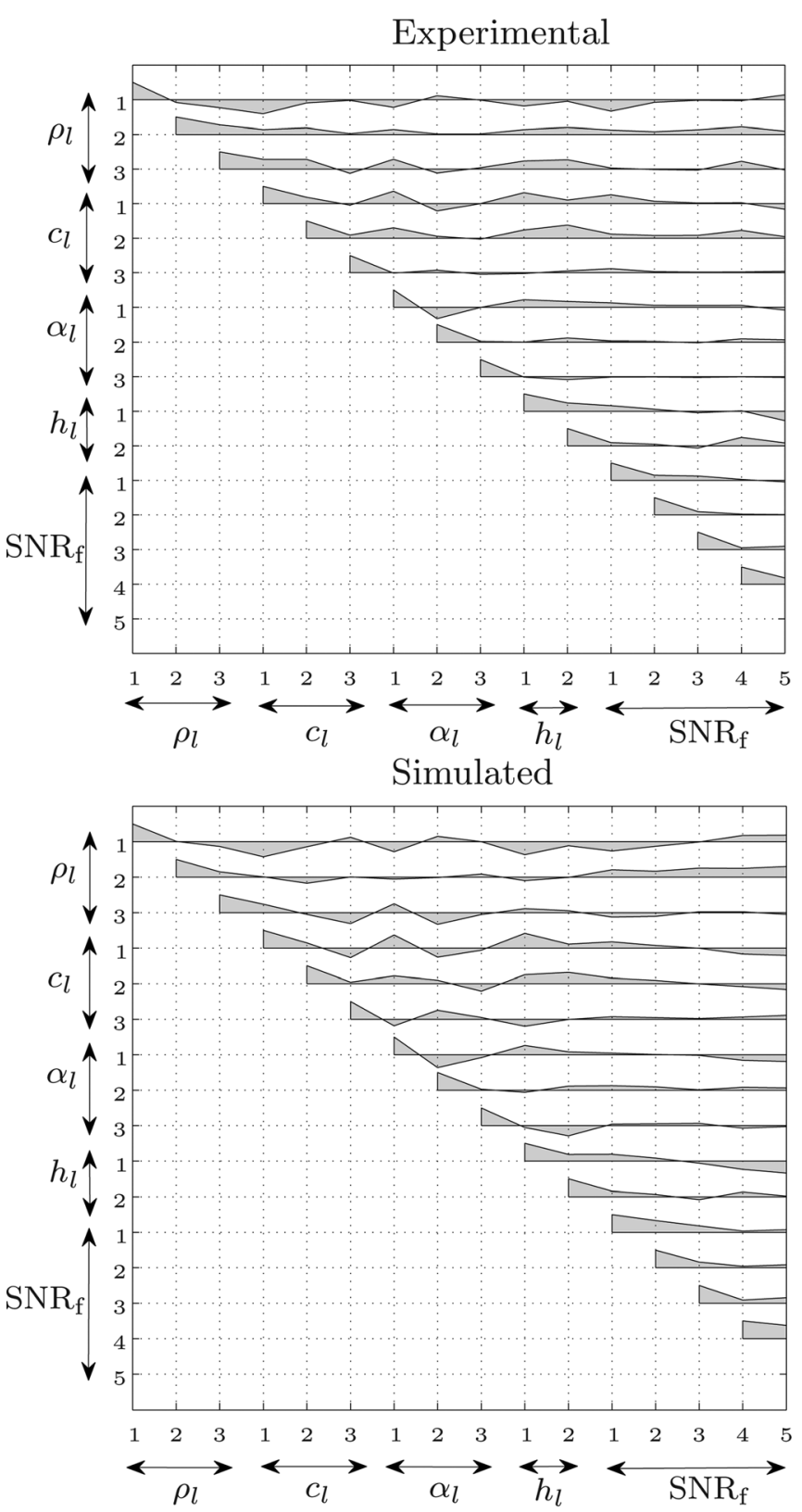

FIG. 10. Parameter correlation matrices from the inversion of measured (top) and simulated (bottom) data with wind speeds of $\sim 20-22$ and 15 kts, respectively. For simplicity, only the upper triangle of the symmetric matrix is plotted. 

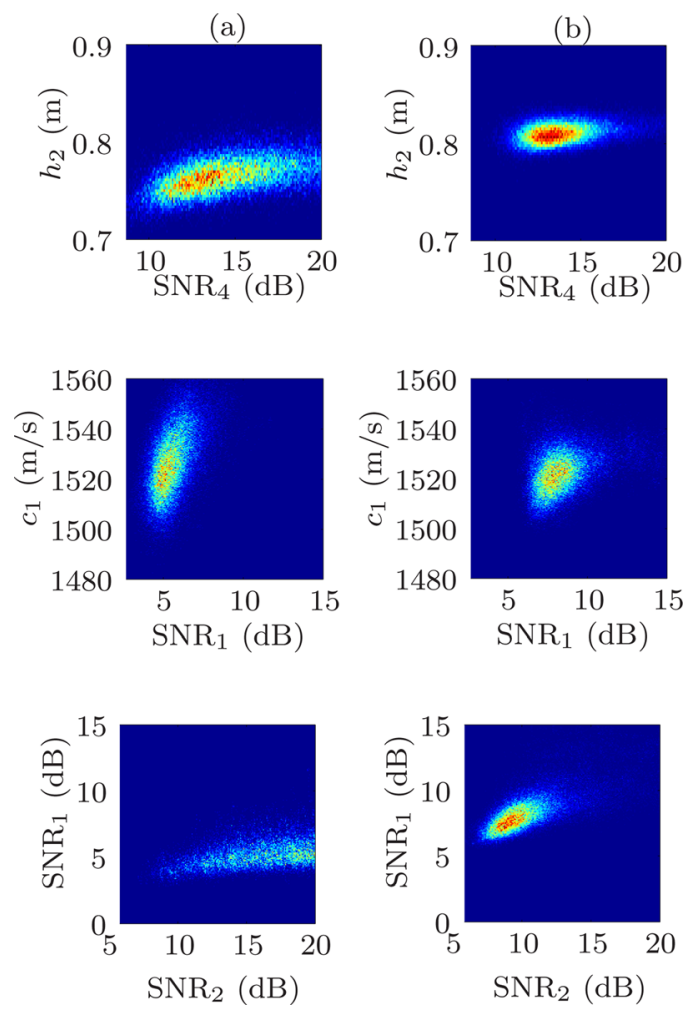

FIG. 11. (Color online) Selected joint marginal PPDs derived from the Bayesian inversion of (a) the experimental data and (b) the simulated data at $V_{s}=15 \mathrm{kts}$.

respectively. On the other hand, the correlations between SNR and attenuations are weaker (smaller than 0.37) in both simulated and experimental cases. It is interesting to see similar correlation patterns when comparing the modeled $\left(V_{s}=15 \mathrm{kts}\right)$ and the measured cases, in particular those involving parameters of layer 1 as well as the correlations between SNRs. The parameter correlations obtained from inversion of simulated data with $V_{s}=10$ and $5 \mathrm{kts}$ (not

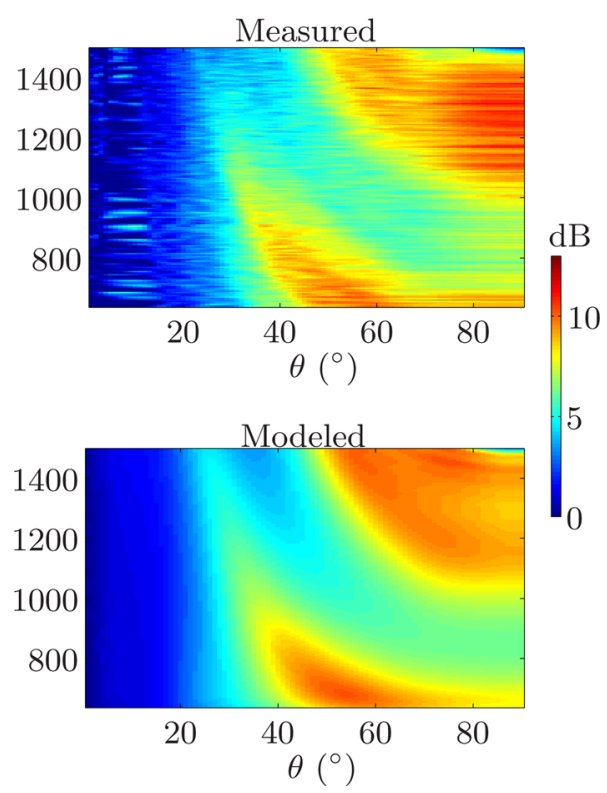

FIG. 12. (Color online) BL computed from measured data (top) and from the forward model evaluated at $\mathbf{m}_{\mathrm{MAP}}$ (bottom). shown in this paper) gradually differ from Fig. 10 as the wind speed decreases.

Examination of joint marginal PPDs in Fig. 11 also reveals strong dependencies between parameters. In this figure, the left column shows the marginals derived from the Bayesian inversion of the experimental data, while the right column corresponds to similar results obtained from the simulated data at $V_{s}=15 \mathrm{kts}$. The exact values of the SNRs may be difficult to match between experiment and simulation because the true level of isotropic noise is unknown in the experimental data. Nevertheless, these joint PPDs demonstrate that fixing the SNR to an arbitrary value or assuming infinite SNR could lead to biased estimates of the geoacoustic parameters.

Finally, using the MAP model obtained from measured data, the bottom loss was computed at frequencies from 600 to $1500 \mathrm{~Hz}$ and angles from $0^{\circ}$ to $90^{\circ}$, as a test of the capability of the MAP model to match data at frequencies and angles other than those used for the inversion. The result is presented in Fig. 12 compared to the experimental bottom loss, and both plots share similar characteristics in the critical angle, location of the main fringes, and loss levels.

\section{CONCLUSIONS}

The results in this paper indicate significant potential for the use of ambient noise for geoacoustic inversion. The proposed method resolves geoacoustic profiles quite well under typical wind-speed conditions, it is inexpensive, unobtrusive, and with low environmental impact.

Consideration of the SNR as a free parameter was found to be a critical step in the inversion. It is evident that low SNR tends to smooth the small-scale details in the bottom loss curves, resulting in loss of information in the data about geoacoustic parameters of deeper layers. This suggests an improvement of data quality with wind speed, up to a point in which rough weather conditions would prevent the establishment of a large (infinite) layer of surface sources as required by the theory. Extremely high wind speeds would probably result in range- and azimuth-dependent distributions of surface sources, which is a topic that has not been explored yet.

One important characteristic of the proposed method is that specific prior knowledge of the SNR is not required, and uniform (non-informative) prior distributions are sufficient for the inversion. The estimated frequency-dependent SNR for the simulated data was in agreement with the theoretical model, but this should not be interpreted as a way to estimate the wind speed when working with experimental data because other factors such as the frequency-dependent sensitivity of the array elements, sensor pre-amplifiers and accuracy of the recording system have a cumulative effect on what is considered the isotropic noise level.

The three examples using simulated data suggest that good conditions for this approach require SNR generally greater than zero. Inversions at $V_{s}=10 \mathrm{kts}$ exhibit tight distributions, and in this case, the theoretical SNRs are $\mathrm{SNR}_{1}=-0.2, \quad \mathrm{SNR}_{2}=1.2, \quad \mathrm{SNR}_{3}=2.7, \quad \mathrm{SNR}_{4}=4.3$, and $\mathrm{SNR}_{5}=5.0 \mathrm{~dB}$. As the $\mathrm{SNR}$ increases, the marginal 
distributions become more compact until the SNR terms become negligible compared to signal terms.

The Bayesian approach quantifies how well the various geoacoustic parameters are resolved as a function of depth and wind speed. For example, for deeper layers and low wind speed the parameters have wide non-informative distributions, indicating no resolution of geoacoustic structure.

Statistical tests of residuals, as well as similar marginal distributions and parameter correlations obtained from experimental $\left(V_{s} \sim 20 \mathrm{kts}\right)$ and simulated $\left(V_{s}=15 \mathrm{kts}\right.$ and $\left.V_{s}=10 \mathrm{kts}\right)$ data, suggest that the forward model can accurately represent the ambient noise field recorded at the VLA. There is good agreement between the parameters obtained from ambient noise data in this paper and a previous inversion using active-source data: In both cases, similar marginal probability profiles with tight distributions for sound speeds and layer thicknesses were observed. The experimental marginal PPDs also agree reasonably well with core samples to $\sim 1.5$-m depth. Discrepancies in density between the core and the active and passive inversions below $\sim 1.5 \mathrm{~m}$ could suggest a problem with the core sample.

The power of the method presented in this work to resolve layers is strongly dependent on the sensitivity of the BL to changes in the layering structure. It is expected that by inverting data mostly at low frequencies, deeper and coarser sediment profiles would be found as opposed to inverting high frequency data, which would provide finer structure at the top layers before the frequency-dependent attenuation becomes a limiting factor in resolving deeper layers. Following this argument, it is advantageous to include a wide range of frequencies to increase the information content on the data.

The inversions presented in this paper take advantage of a moored array for which several unknowns such as array vertical displacement, tilting, and positioning errors are not likely to play a significant role in introducing artifacts. Although these potential challenges must be considered with non-fixed configurations, previous work on the passive fathometer ${ }^{11}$ indicates that the ambient noise covariance matrix can also be accurately estimated from data collected in a slowly drifting array.

\section{ACKNOWLEDGMENTS}

The authors gratefully acknowledge the support of the Office of Naval Research postdoctoral fellowship and the Ocean Acoustics Program (ONR-OA Code 32). We also would also like to acknowledge the NATO Undersea Research Centre for providing the MAPEX2000bis data and Dr. Charles W. Holland for providing the core measurements.

${ }^{1}$ C. H. Harrison and D. G. Simons, "Geoacoustic inversion of ambient noise: A simple method,” J. Acoust. Soc. Am. 112, 1377-1389 (2002).
${ }^{2}$ M. Siderius, C. H. Harrison, and M. B. Porter, "A passive fathometer technique for imaging seabed layering using ambient noise," J. Acoust. Soc. Am. 120, 1315-1323 (2006).

${ }^{3} \mathrm{M}$. Siderius and C. H. Harrison, "High-frequency geoacoustic inversion of ambient noise data using short arrays," AIP Conf. Proc. 728, 22-31 (2004).

4J. I. Arvelo, "Robustness and constraints of ambient noise inversion," J. Acoust. Soc. Am. 123, 679-686 (2008).

${ }^{5}$ J. Dettmer, S. E. Dosso, M. K. Prior, C. H. Harrison, and N. R. Chapman, "Geoacoustic inversion of ambient noise reflection-loss data," in Proceedings of the Seventh European Conference on Underwater Acoustics, Delft, The Netherlands (2004), pp. 511-516.

${ }^{6}$ J. Dettmer, C. W. Holland, and S. E. Dosso, "Analyzing lateral seabed variability with Bayesian inference of seabed reflection data," J. Acoust. Soc. Am. 126, 56-69 (2009).

${ }^{7}$ W. A. Kuperman and F. Ingenito, "Spatial correlation of surface generated noise in a stratified ocean," J. Acoust. Soc. Am. 67, 1988-1996 (1980).

${ }^{8}$ C. H. Harrison, "Formulas for ambient noise level and coherence," J. Acoust. Soc. Am. 99, 2055-2066 (1996).

${ }^{9}$ H. Schmidt, OASES version 3.1 User guide and reference manual; http:// acoustics.mit.edu/faculty/henrik/oases.html (Last viewed 08/02/2011).

${ }^{10} \mathrm{~F}$. Ingenito and S. N. Wolf, "Site dependence of wind-dominated ambient noise in shallow water," J. Acoust. Soc. Am. 85, 141-145 (1988).

${ }^{11}$ M. Siderius, H. Song, P. Gerstoft, W. S. Hodgkiss, P. Hursky, and C. H. Harrison, "Adaptive passive fathometer processing," J. Acoust. Soc. Am. 127, 2193-2200 (2010).

${ }^{12}$ S. E. Dosso and P. L. Nielsen, "Quantifying uncertainty in geoacoustic inversion. II. Application to broadband shallow water data," J. Acoust. Soc. Am. 111, 143-159 (2002).

${ }^{13}$ C. F. Huang, P. Gerstoft, and W. S. Hodgkiss, "Uncertainty analysis in matched-field geoacoustic inversions," J. Acoust. Soc. Am. 119, 197-207 (2006).

${ }^{14}$ S. E. Dosso and J. Dettmer, "Bayesian matched-field geoacoustic inversion," IOP J. Inv. Problems 27, 23 (2011).

${ }^{15}$ J. Dettmer, C. W. Holland, and S. E. Dosso, "Joint time/frequency-domain inversion of reflection data for seabed geoacoustic profiles and uncertainties," J. Acoust. Soc. Am. 123, 1306-1317 (2008).

${ }^{16}$ S. Kirkpatrick, C. D. Gelatt, and M. P. Vecchi, "Optimization by simulated annealing," Science, New Ser. 220, 671-680 (1983).

${ }^{17} \mathrm{P}$. Gerstoft and Z. Michalopoulou, "Global optimization in matched field inversion," in 4th European Conference on Underwater Acoustics (Italian National Research Council), Rome, (2000) pp. 27-32.

${ }^{18}$ A. Basu and L. N. Frazer, "Rapid determination of the critical temperature in simulated annealing inversion," Science 21, 1409-1412 (1990).

${ }^{19}$ N. Metropolis, A. W. Rosenbluth, M. N. Rosenbluth, A. H. Teller, and E. Teller, "Equations of state calculations by fast computing machines," J. Chem. Phys. 21, 1087-1092 (1953).

${ }^{20}$ W. K. Hastings, "Monte Carlo sampling methods using markov chains and their applications," Biometrika 57, 97-109 (1970).

${ }^{21}$ S. E. Dosso and M. J. Wilmut, "Uncertainty estimation in simultaneous Bayesian tracking and environmental inversion," J. Acoust. Soc. Am. 124, 82-97 (2008).

${ }^{22}$ M. D. Collins and L. Fishman, "Efficient navigation of parameter landscapes," J. Acoust. Soc. Am. 98, 1637-1944 (1995).

${ }^{23}$ F. B. Jensen, W. A. Kuperman, M. B. Porter, and H. Schmidt, Computational Ocean Acoustics (American Institute of Physics, New York, 1994).

${ }^{24} \mathrm{M}$. Schulkin, "Sea surface loss in surface ducts and shallow water: a historical perspective," in Proceedings of the IEEE Oceans'86 Conference, Washington, D.C. (1986), pp. 325-331.

${ }^{25}$ S. E. Dosso, P. L. Nielsen, and M. J. Wilmut, "Data error covariance in matched-field geoacoustic inversion," J. Acoust. Soc. Am. 119, 208-219 (2006).

${ }^{26}$ J. Dettmer, S. E. Dosso, and C. W. Holland, "Model selection and Bayesian inference for high resolution seabed reection inversion," J. Acoust. Soc. Am. 125, 706-716 (2009).

${ }^{27}$ D. R. Jackson and M. D. Richardson, High-Frequency Seafloor Acoustics (Springer Science and Business Media, New York, 2007). 